\title{
Guest editorial-second part of special issue on cloud computing
}

\author{
Dilma Da Silva $\cdot$ Xu Dongyan $\cdot$ Dan Reed
}

Published online: 5 November 2011

(C) The Brazilian Computer Society 2011

In this issue we continue with our selection of papers highlighting innovative work on cloud computing. This time we cover naming and resource location, virtual machine placement, scheduling work on hybrid clouds, and authorization management.

In "A XRI Naming System for Dynamic and Federated Clouds: a Performance Analysis", Antonio Celesti, Massimo Villari, and Antonio Puliafito investigate the challenges in providing a common naming and location service for identifying virtual resources residing in different operating systems. They describe and evaluate an approach for extending the OpenXRI architecture to provide a front-end for cloud name space management.

The paper "HCOC: a cost optimization algorithm for workflow scheduling in hybrid clouds", by Luiz Fernando Bittencourt and Edmundo Roberto Mauro Madeira, looks at the problem of dynamically extending private clouds by adding resources from public clouds. They propose an algorithm to decide when to lease resources from a public cloud in order to execute a workflow within a specified execution time.

D. Da Silva $(\bowtie)$

IBM TJ Watson Research Center, Yorktown Heights, NY, USA

e-mail: dilmasilva@us.ibm.com

X. Dongyan

Department of Computer Science and Department of Electrical and Computing Engineering, Purdue University, West Lafayette, USA

e-mail: dxu@cs.purdue.edu

D. Reed

Technology Policy and Strategy and Leader of the eXtreme

Computing Group at Microsoft Research, Seattle, USA

e-mail: daniel.Reed@microsoft.com
Caroline Shouraboura and Pavel Bleher attack the problem of placing virtual machines in physical hosts so that low-latency and high-durability are achieved. Their paper "Placement of applications in computing clouds using Voronoi diagrams" proposes a novel data structure and algorithm that leverages ideas from computational geometry for modeling global cloud resources.

Finally, "Toward role-based provisioning and access control for infrastructure as a service (IaaS)", by Dongwan Shin, Hakan Akkan, and William R. Claycomb, proposes a novel approach for access control in the cloud. They introduce a domain-based, decentralized framework for provisioning and managing users and virtualized resources that satisfy requirements by enterprise customers not currently addressed by the main providers of Infrastructure-asa-Service.

As we did with the selection of cloud computing papers in the previous issue, we would like to thank the authors for their contributions and the anonymous reviewers for their expert guidance in improving the papers. We are grateful to JISA's Editors-in-Chief and their associated editorial team for their excellent support.

Sincerely,

Guest Editors 